\title{
Modified Transition For Substrate Integrated Waveguide(SIW) Structures
}

\author{
Amirhossein ASKARIAN $^{1}$, Gholamreza MORADI ${ }^{2}$ \\ ${ }^{1}$ ASKARIAN, Amirhossein, Electrical Engineering Faculty of Amirkabir University of \\ Technology (Tehran Polytechnic), Tehran, Iran, Askarian.amirhossein@aut.ac.ir. \\ ${ }^{2}$ MORADI ,Gholamreza, Associated professor in Electrical Engineering Faculty of \\ Amirkabir University of Technology (Tehran Polytechnic), Tehran, Iran,Ghmoradi@aut.ac.ir.
}

\begin{abstract}
In this paper a new X-band SIW to Microstrip transition based on SIW structure is proposed. The recent transition has better performance in terms of return loss than those of in the traditional Microstrip tapers transition. Chebyshev multi-section matching transformer is utilized in order to inter-stage network between Microstrip and SIW, whereof it is the most optimum and efficient in matching networks theory. The $5^{\text {th }}$-order Chebyshev polynomial is employed to get about $30 \mathrm{~dB}$ return loss. For this purpose, curve-fitting technique is utilized to obtain a mathematical function based on Chebyshev polynomial which presents the optimum position of vias in SIW transition. In order to validation of theoretical method and results, a full-wave simulation is carried out by CST STUDIO SUITE software.
\end{abstract}

Keywords:

Chebyshev, Curve-fitting, high-Q factor, Permittivity, RWG, SIW, TEM, TE, X-band.

\section{Introduction}

Rectangular Waveguide (RWG) is a low loss, high-Q factor and wideband waveguide for realizing microwave and millimeter-wave structures and devices. Large dimensions, difficult manufacturing and difficulty to transition to Microstrip transmission lines, are main problems against wide-spread using in microwave applications. Substrate Integrated Waveguide (SIW) is proper alternative for RWG structures. SIW structure first introduced around 1994 [1, 6]. As depicted in Fig. 1, this planar structure consists of one metalized substrate and two rows of metallic vias that act like metallic walls in RWG. Electromagnetic energy is propagated in space between two metallic plates in top and bottom of substrate and two rows of vias. Both advantages of RWG and planar transmission lines are integrated in SIW, among them, easy to manufacture, simple transition to Microstrip, small size respect to RWG, low-loss, and highbandwidth versus Microstrip lines. Therefore because of the mentioned outstanding features of SIW, it can be a good trade-off between RWG and planar microwave structures. Microstrip tapered lines are used as impedance matching networks in SIW structure but as mentioned in [1], for SIWs with characteristic impedance greater than $5 \Omega$, these kind of transitions cannot 
be efficient networks, thus a new transition based on SIW structure is proposed in this paper. Since impedance matching networks are fundamental problem in microwave and millimeters wave, this paper concentrates on finding precise and effective impedance matching network in microwave frequencies. Although, in [1] some new SIW transitions have been presented but seems, more optimizations and improvements in performance are required.

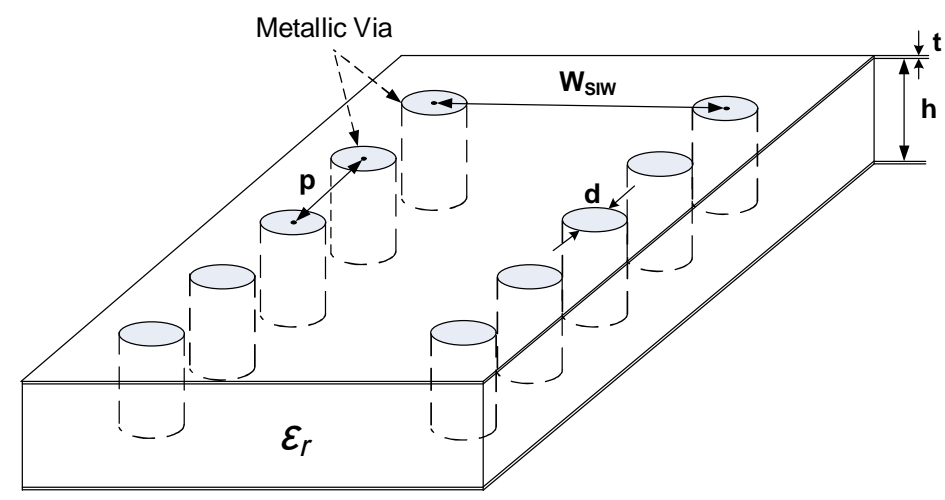

Fig. 1: A typical SIW structure

\section{Multisection Impedance Matching}

\subsection{Chebyshev Multisection Impedance Matching}

Multi-section transformer as shown in Fig. 2 consists of $N$ equal-length (usually quarterwave) section of transmission lines. According to theory of small reflections, the overall reflection coefficient can be approximated as [2-3]

$\Gamma(\theta)=\Gamma_{0}+\Gamma_{1} e^{-2 j \theta}+\Gamma_{2} e^{-4 j \theta}+\cdots+\Gamma_{N} e^{-2 j N \theta}$.

By properly selecting the partial reflection coefficient $\Gamma_{\mathrm{n}}$ and also using enough sections $\mathrm{N}$, it is possible to synthesize any desired reflection coefficient response as a function of frequency. This work uses Chebyshev Multi-section matching transformer whereas in contrast with the binominal matching transformer, it is more efficient in bandwidth at the expense of passband ripple than binominal matching transformer for a given number of sections. General form of Chebyshev polynomial can be written as recurrence formula [2-3]

$T_{N}(x)=2 x T_{N-1}(x)-T_{N-2}(x)$

With substituting $x=\cos (\theta)$ for $|x|<1$ and map $\theta_{m}$ to $x=1$ and $\pi-\theta_{m}$ to $x=-1$ where $\theta_{m}$ and $\pi-\theta_{m}$ are the lower and upper edges of the passband, Chebyshev polynomial becomes:

$T_{N}\left(\sec \theta_{m} \cos \theta\right)=\cos \left(N\left[\cos ^{-1}\left(\sec \theta_{m} \cos \theta\right)\right]\right)$. 


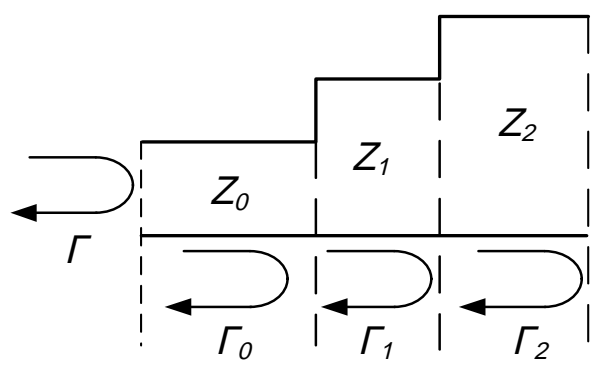

Fig. 2: Multi-Section Impedance transformation

Here, the $5^{\text {th }}$-order of (3) is used for designing SIW taper so

$T_{m}\left(\sec \theta_{m} \cos \theta\right)=\sec ^{5} \theta_{m} \cos 5 \theta-5 \sec ^{2} \theta_{m}\left(1-\sec ^{2} \theta_{m}\right) \cos 3 \theta+5 \sec \theta_{m}\left(2 \sec ^{4} \theta_{m}-3 \sec ^{2} \theta_{m}+1\right) \cos \theta$

where $\theta_{m}=33.3^{\circ}$ corresponding to $\Gamma_{m}=30 d B$ and bandwidth for TEM wave is about $126 \%$. Fig. 3 shows reflection coefficient respect to $\theta$ in radian.

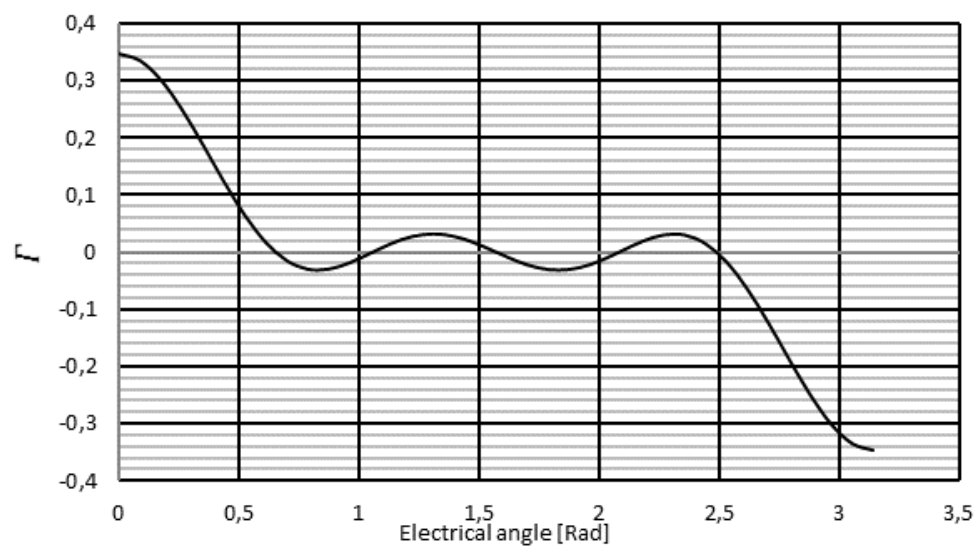

Fig. 3:Reflection coefficient 5th-order Chebyshev multisession impedance transformer respect to electrical angle.

\subsection{Generalization Multisection Impedance Matching to SIW}

The main purpose of this section is, generalization of achievement that are obtained in pervious section from TEM wave to TE mode, so for this purpose, relation of characteristic impedance in RWG structure for dominate mode $\mathrm{TE}_{10}$ is given in (5) [4], since RWG and SIW are very similar thus it is possible to use (5) for SIW with equivalent dimensions according to (6) and (7), [5]

$Z_{0}=465 \frac{h}{w_{r e c}} \frac{1}{\sqrt{\varepsilon_{r}}} \frac{1}{\sqrt{1-\left(\frac{f_{g}}{f}\right)^{2}}}$ 


$$
\begin{aligned}
& w_{r e c}=w_{S I W}-1.08 \frac{d^{2}}{p}+0.1 \frac{d^{2}}{w_{S W}} \\
& \left(\lambda_{g}\right)_{T E_{10}}=2 \pi / \sqrt{\left(\frac{\varepsilon_{r} \omega^{2}}{c^{2}}\right)-\left(\frac{\pi}{w_{S W}}\right)^{2}}
\end{aligned}
$$

where, $\mathrm{h}$ is thickness of substrate, $w_{\text {reo }}$ and $w_{S I}$ are widths of RWG and SIW respectively, $f_{c}$ is cut-off frequency in X-band that can be considered around 7.8 GHz. $\mathrm{d}$ and $\mathrm{p}$ are diameter and pitch of vias in SIW respectively. Regarding to results in previous section and using (5) and (6), a $5^{\text {th }}$-order multisession Chebyshev for SIW is investigated by curve fitting. As shown in Fig. 4, the computational curve determines position of vias in SIW transition along the length of $5 \lambda_{g} / 4$. Characteristic impedance is gradually varied from $\mathrm{Z}_{\mathrm{SIW}}$ to $\mathrm{Z}_{\mathrm{MS}}$ according to $5^{\text {th }}$-order Chebyshev polynomial.

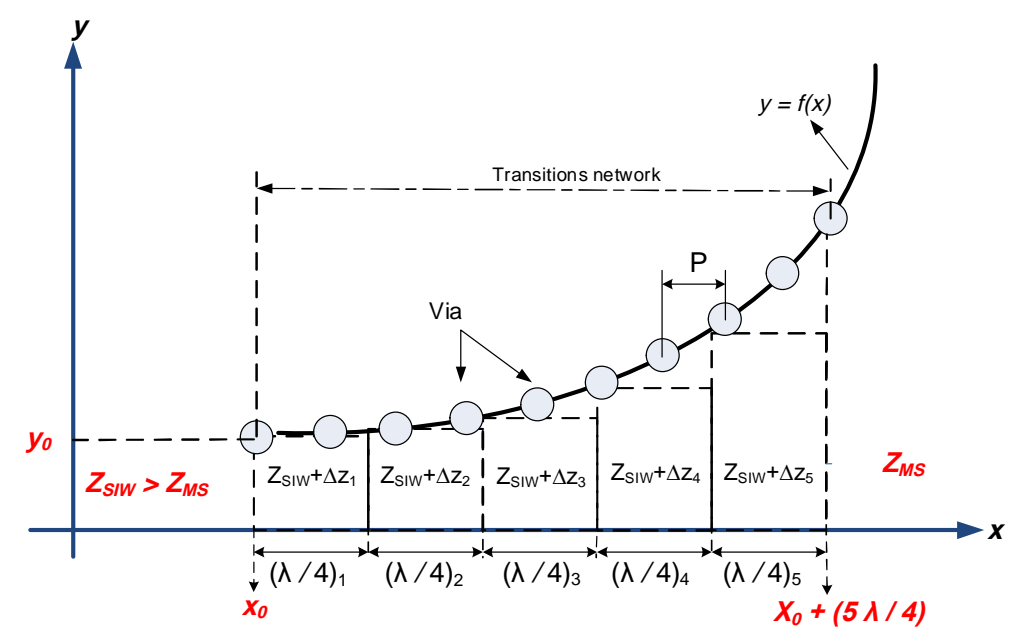

Fig. 4: Computational curve for $Z_{(\text {SIW })}>Z_{(\text {MS) }}$

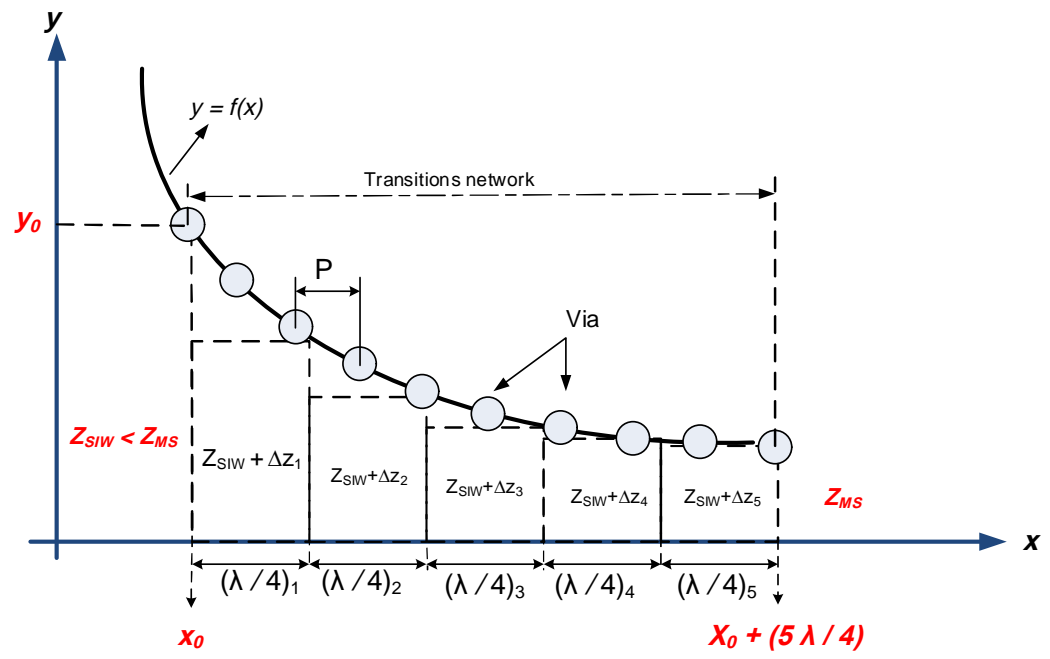

Fig. 5: Computational curve for $Z_{\text {(SIW) }}<Z_{\text {(MS) }}$ 
Characteristic impedance for each structure can be calculated by equation (5) and then according to impedance values, optimum positions of vias can be determined by following Equations:

$$
\begin{aligned}
& y=0.5 \times\left(a\left(\frac{4}{A_{a}}\left(x-x_{0}\right)\right)^{4}+b\left(\frac{4}{A_{a}}\left(x-x_{0}\right)\right)^{2}+c\left(\frac{4}{\lambda_{\theta}}\left(x-x_{0}\right)\right)^{2}+d\left(\frac{4}{A_{g}}\left(x-x_{0}\right)\right)^{2}+e\right) \\
& x=n \frac{\lambda_{g}}{4}+x_{0} \quad 0<n \leq 5 \\
& a=-1.74 \times 10^{-3} e^{-0.054\left(\frac{w_{m s}}{w_{\text {SIW }}}\right)}+1.34 \times 10^{-3} e^{-0.262\left(\frac{w_{\text {MIS }}}{w_{\text {SIW }}}\right)} \\
& b=0.81 \times 10^{-3} e^{0.019\left(\frac{w_{m s}}{w_{S W}}\right)}-0.083 \times 10^{-3} e^{-1.68\left(\frac{w_{m s}}{w_{S I W}}\right)} \\
& c=2.14 \times 10^{-3} e^{0.192\left(\frac{w_{m s}}{w_{S I}}\right)}-0.6 \times 10^{-3} e^{-0.8\left(\frac{w_{m s}}{w_{S I W}}\right)} \\
& d=-5.2 \times 10^{-3} e^{0.051\left(\frac{w_{m s}}{w_{S I W}}\right)}+0.34 \times 10^{-3} e^{-1.8\left(\frac{w_{m s}}{w_{S W}}\right)} \\
& e=0.33 \times 10^{-3} e^{-1.74\left(\frac{w_{m s}}{w_{S W}}\right)}-6.6 \times 10^{-3} e^{-0.16\left(\frac{w_{m s}}{w_{S W}}\right)}
\end{aligned}
$$

Where, $\mathrm{y}$ is transverse distance and $\mathrm{x}$ is the number of quarter wavelength in longitudinal distance. $w_{m s}$ and $w_{S W}$ are width at the two endings of structure (Microstrip and SIW sides respectively) as shown in Fig. 5. The widths ratio $\frac{w_{n n s}}{w_{\text {GIW }}}$, is a function of diameter of vias, according to (9):

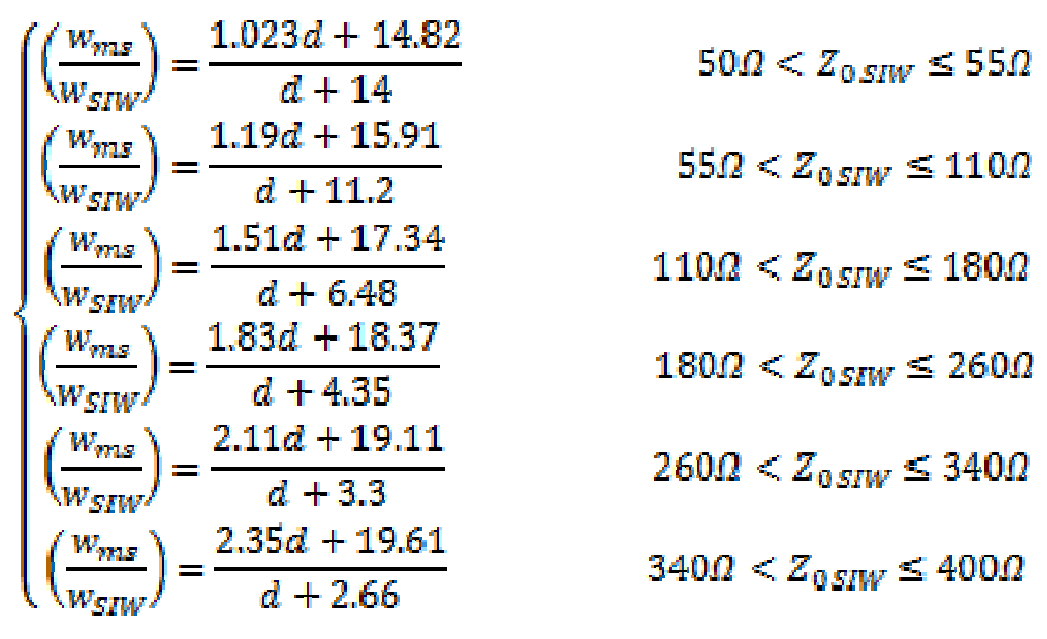

where, $0.1^{m m}<d<4^{m m}$ and $Z_{0 S W}$ is SIW characteristic impedance which is intended to approach $50 \Omega\left(\mathrm{Z}_{m s}\right)$. Fig. 5 shows the corresponding curve of sample SIW transition. 


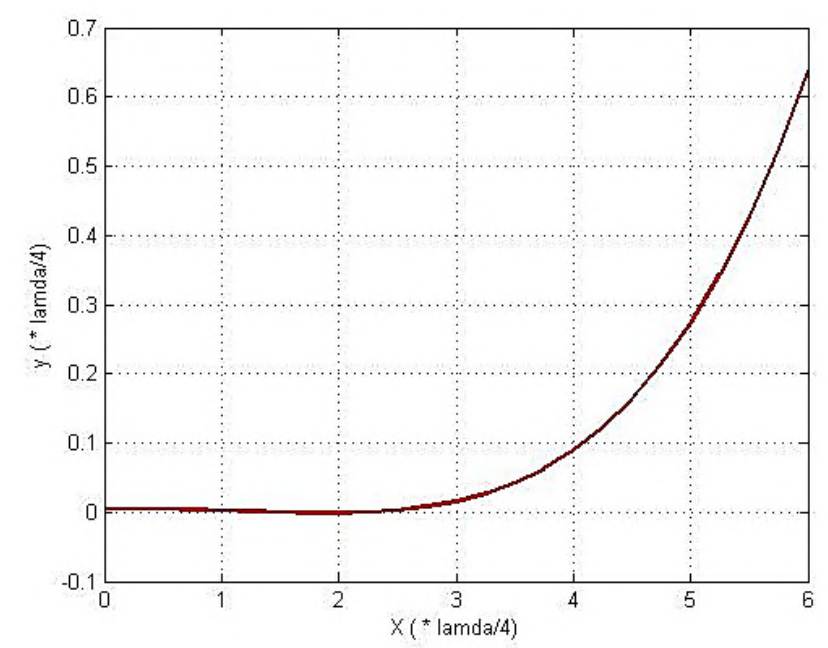

Fig. 6: Corresponding curve for sample X-band SIW structure

Since (8) and (9) are driven from typical structure in X-band, so it is essential to investigate the impacts of other structure's parameters by means of sensitivity analysis.

According to equation (5), characteristic impedance of SIW is a function of several parameters like thickness and permittivity of substrate, pitch and diameter of vias so various types of SIW structure with different parameters lead to various characteristic impedance then some reformation are needed as a correction factors in the equation (9). Since equation (9) presents distinct relations for various amount of $Z_{0 \text { SIW }}$, correction coefficient $\Delta z_{o}$ should multiply with $Z_{0 S I W}$; therefor, for more accuracy $Z_{0 S W} \times \delta Z_{0 S W}$ must be inserted into equation (9).

To obtain $\delta Z_{0 S T W}$, sensitivity analysis should be carried out as equation (10) indicates, sensitivity analysis reveals the characteristic impedance's changes respect to thickness of substrate h, permittivity of substrate $\varepsilon_{r}$ and pitch of vias $\mathrm{P},[8]$.

$\delta Z_{0 S T}=\frac{\left|\Delta Z_{0 S T}\right|_{\max }}{Z_{0 S W}}=\left|\frac{\Delta h}{h} S_{h}^{z_{0}}\right|+\left|\frac{\Delta \varepsilon_{r}}{\varepsilon_{r}} S_{z_{r}}^{z_{0}}\right|+\left|\frac{\Delta p}{p} S_{p}^{z_{0}}\right|+\left|\frac{\Delta p}{d} S_{d i}^{z_{0}}\right|$

$S_{h}^{z_{0}}=\frac{\partial Z_{0}}{\partial h} \frac{h}{Z_{0}}$

$S_{\varepsilon_{r}}^{z_{0}}=\frac{\partial Z_{0}}{\partial \varepsilon_{r}} \frac{\varepsilon_{r}}{Z_{\mathrm{a}}}$

$S_{p}^{Z_{0}}=\frac{\partial Z_{0}}{\partial p} \frac{p}{Z_{\mathrm{a}}}$ 
$S_{d}^{z_{0}}=\frac{\partial Z_{0}}{\partial d} \frac{d}{Z_{0}}$

where, $S_{h}^{\Sigma_{0}}, S_{z_{q}}^{z_{0}}, S_{p}^{z_{0}}$, and $S_{d}^{z_{0}}$ are proper normalized sensitivity function. Figs. 6-8 show normalized changes of parameters (permittivity, dielectric thickness and via pitch respectively); note that, normalized impedance is $50 \Omega$. This analysis helps speculate effects of parameter variations in the characteristic impedance amounts. Using the results of (9), positions of vias can be optimized for wide variety of structural parameters. Typical dimensions of SIW in X-band are indicated in Table I. In mentioned structure, from (5) and

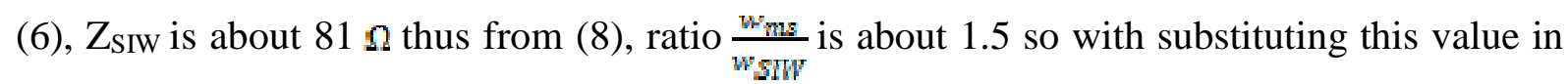
(7), relative curve can be calculated. In this example, in sensitivity analysis, if permittivity changes about $65 \%$ of the typical value, normalized characteristics impedance will be changed about $10 \%$, so it should be considered in using (9).

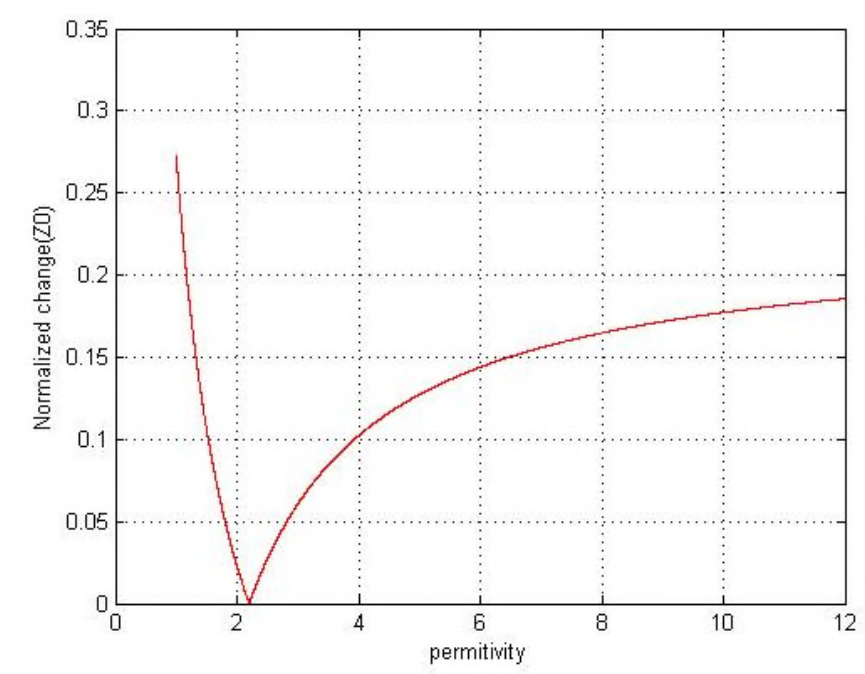

Fig. 7: Normalized change of $Z_{0}$ respect to permittivity. 


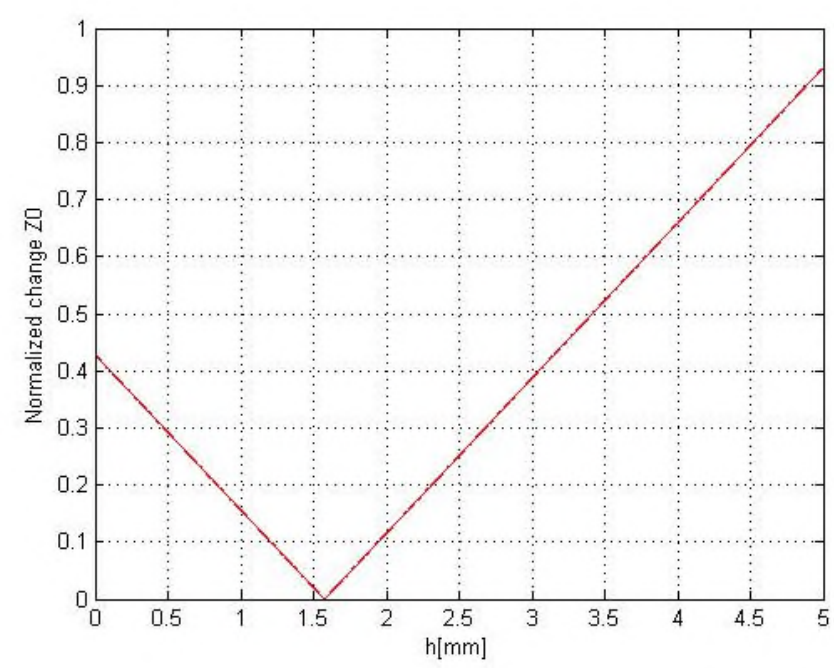

Fig. 8: Normalized change of $\mathrm{Z} 0$ respect to dielectric thickness

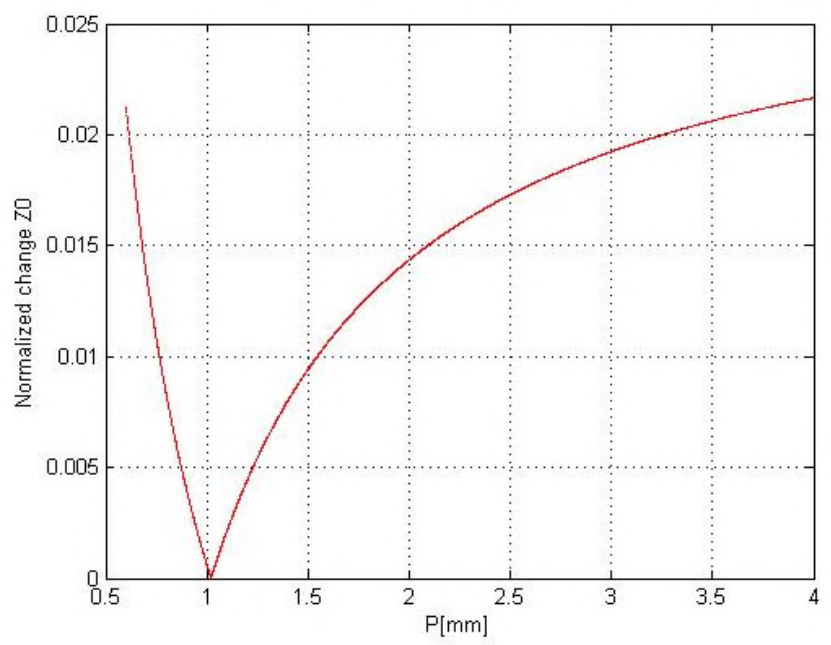

Fig. 9 : Normalized change of $Z_{0}$ respect to pitch of Via.

\section{Simulation and analysis by CST STUDIO SUITE}

In order to investigate and validate the proposed algorithm and formula, full-wave simulation is carried out by means of CST STUDIO SUITE full-wave simulator in frequency range of 8 to $16 \mathrm{GHz}$. Two transition structure is presented here. The first one is based on traditional taper-out transition as has been shown in Fig. 10. Optimization has been utilized in order to obtaining best parameters which are shown in table 1. Fig. 11 illustrates both return loss and insertion loss. Having surveyed the results, it is obvious the best return loss is about $22 \mathrm{~dB}$ and insertion loss is better than $0.5 \mathrm{~dB}$.

The second one (New transition) is according to combination of Microstrip and SIW transition which has been achieved better results in terms of return loss as well as insertion loss. With a brief glance at the Fig. 12 it is so explicit that deviation some vias in accordance with Chebyshev polynomial which has been introduced in equation (8). In accordance with 
Bulletin de la Société Royale des Sciences de Liège, Vol. 85, 2016, p. 203 - 214

Fig. 13, the results significantly improved so that best return loss is $30 \mathrm{~dB}$ while insertion loss is better than $0.75 \mathrm{~dB}$.

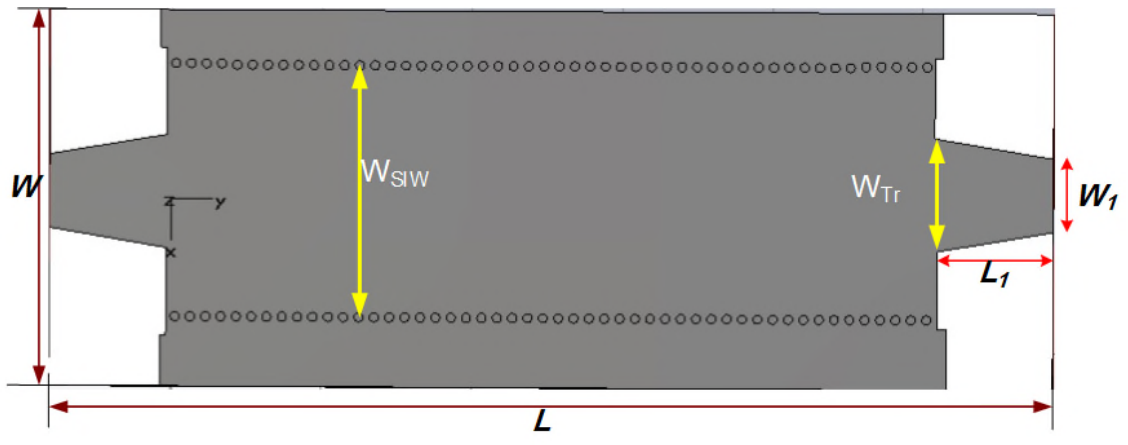

Fig. 10: Back to back SIW structure with traditional Microstrip transition.

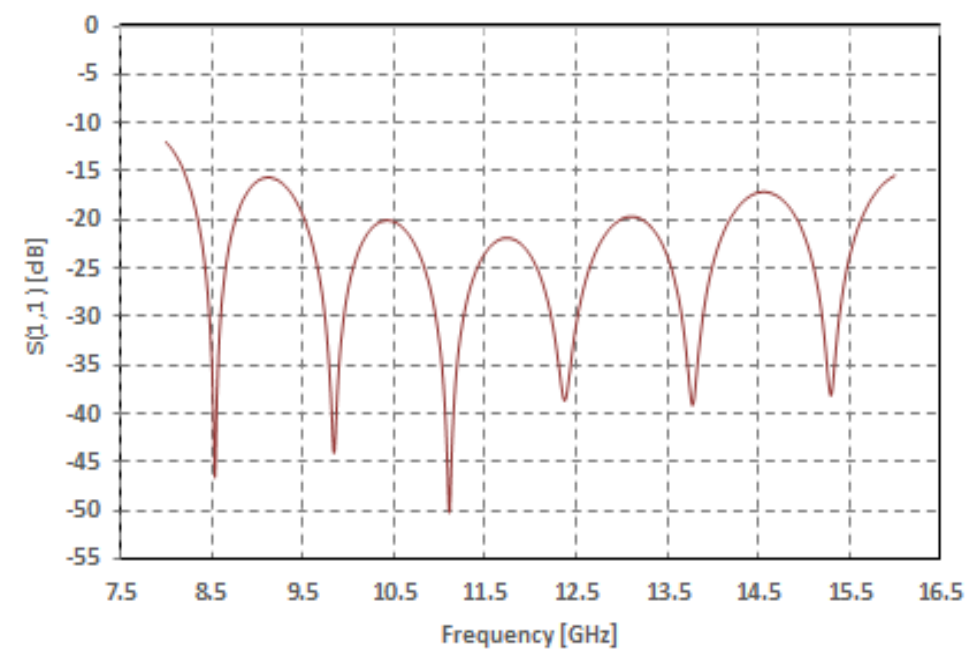

(a)

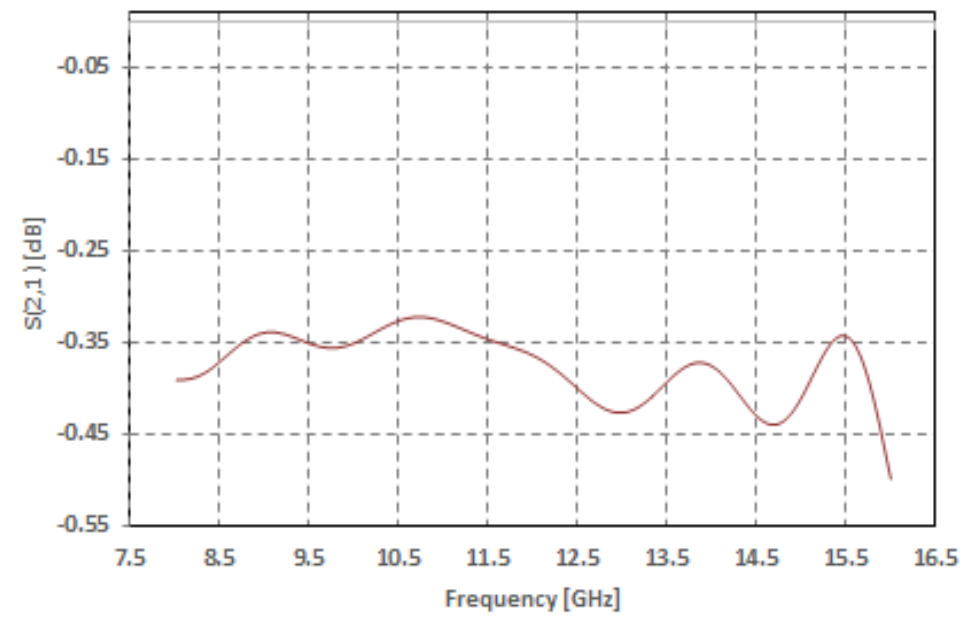

(b)

Fig. 11: S-parameters of traditional transition (a) Return loss (b) Insertion loss 
Bulletin de la Société Royale des Sciences de Liège, Vol. 85, 2016, p. 203 - 214

Table 1: Optimized Dimensions of SIW With traditional Transition

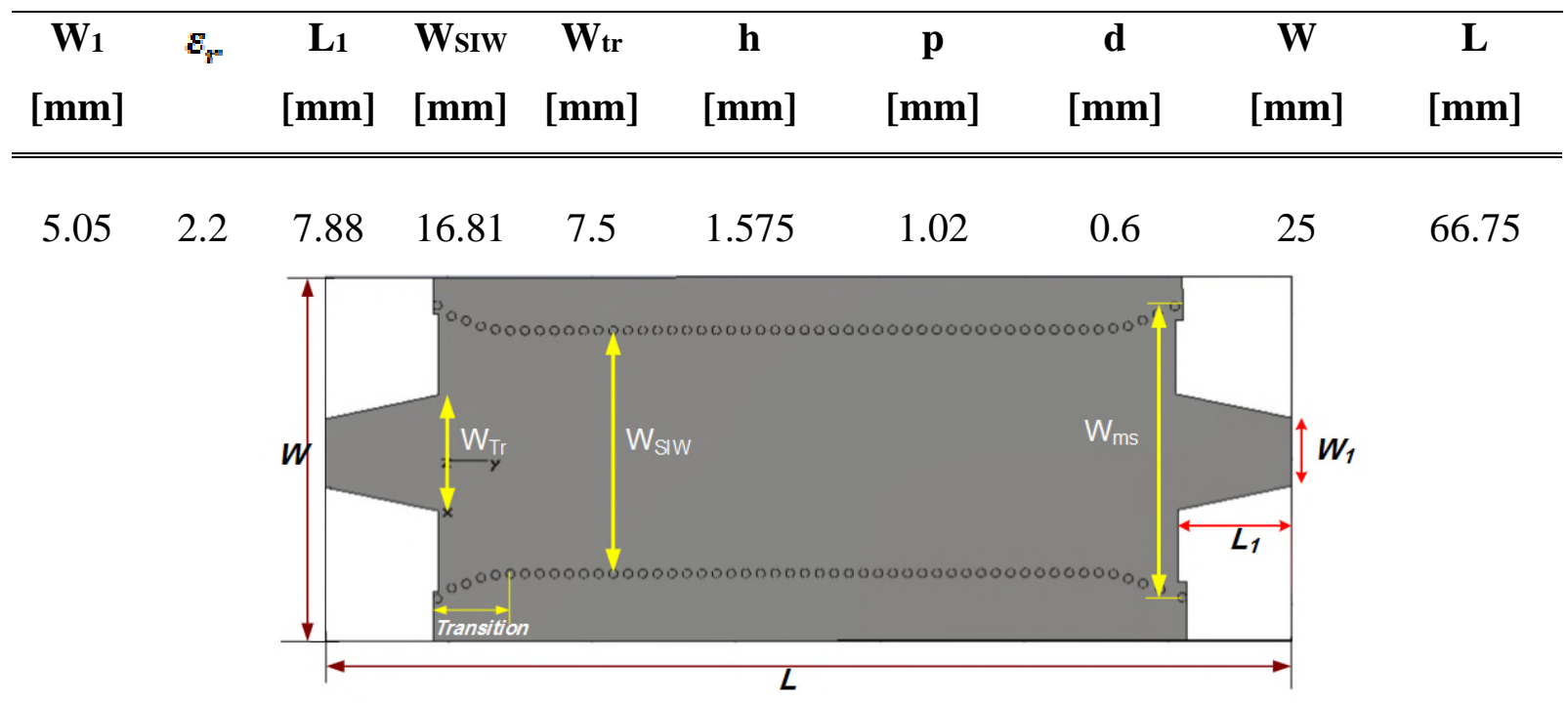

Fig. 12 :Back to back SIW structure with new transition.

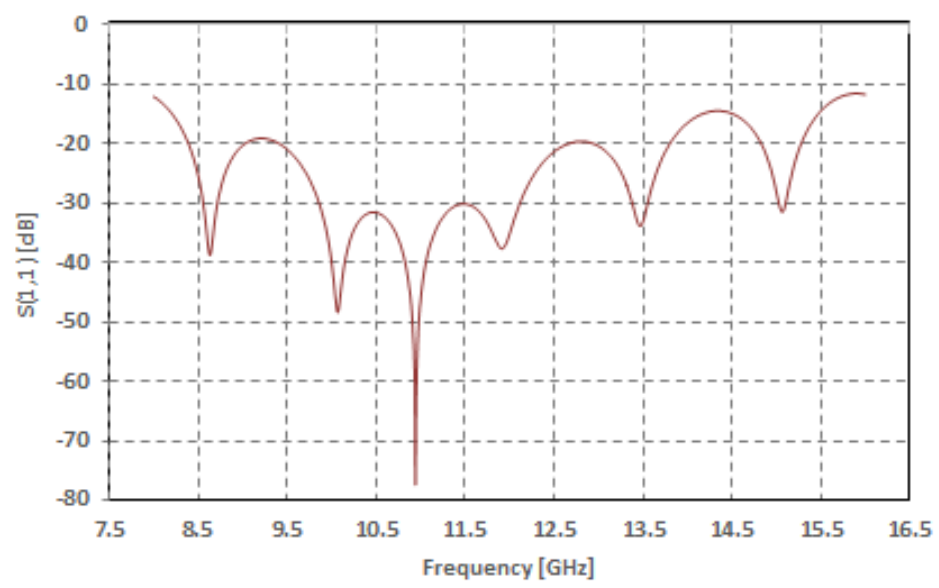

(a)

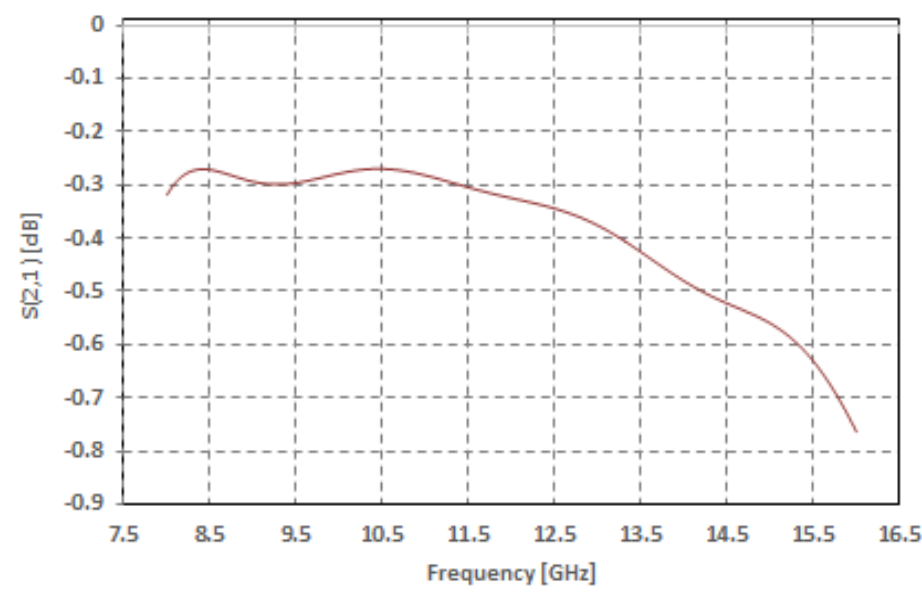

(b)

Fig. 13: S-parameters of new transition (a) Return loss (b) Insertion loss 
Having scrutinized the results, there are proper agreement between theoretical impedance matching analyses which has been done based on TEM mode (equation (8)) and full-wave simulation which is based on SIW structure in $\mathrm{TE}_{10}$ mode.

Table 2: Optimized Dimensions of SIW With New Transition

\begin{tabular}{ccccccccccc}
\hline $\begin{array}{c}\mathbf{W}_{1} \\
{[\mathbf{m m}]}\end{array}$ & $\varepsilon_{\boldsymbol{r}}$ & $\begin{array}{c}\mathbf{L}_{1} \\
{[\mathbf{m m}]}\end{array}$ & $\begin{array}{c}\mathbf{W}_{\text {SIW }} \\
{[\mathbf{m m}]}\end{array}$ & $\begin{array}{c}\mathbf{W}_{\mathbf{m s}} \\
{[\mathbf{m m}]}\end{array}$ & $\begin{array}{c}\mathbf{W}_{\mathbf{t r}} \\
{[\mathbf{m m}]}\end{array}$ & $\begin{array}{c}\mathbf{h} \\
{[\mathbf{m m}]}\end{array}$ & $\begin{array}{c}\mathbf{p} \\
{[\mathbf{m m}]}\end{array}$ & $\begin{array}{c}\mathbf{d} \\
{[\mathbf{m m}]}\end{array}$ & $\begin{array}{c}\mathbf{W} \\
{[\mathbf{m m}]}\end{array}$ & $\begin{array}{c}\mathbf{L} \\
{[\mathbf{m m}]}\end{array}$ \\
\hline \\
\hline 5.05 & 2.2 & 8.04 & 16.81 & 20.2 & 7.98 & 1.575 & 1.02 & 0.6 & 25 & 67.03
\end{tabular}

\section{Conclusion}

Here, an X-band SIW to Microstrip transition based on SIW structure has been proposed. Whereas Chebyshev impedance matching network is more bandwidth efficient than other types such as binomial matching network. The $5^{\text {th }}$-order Chebyshev polynomial is being used for determining optimum positions of vias in this kind of transition. To obtain the guide line for placing vias the curve fitting technique is utilized. Since SIW has less loss than Microstrip lines, thus this type of transition is more efficient and optimum in loss. Outstanding feature of this impedance network is easy to manufacture, so that it is realized with some deviations in path of vias in one or both side(s) of SIW rows. All in all, a validation and generalization of quarter-wave Chebyshev impedance matching network to TE mode is carried out, since this method used to apply for TEM (or quasi-TEM) structures.

\section{References}

[1] E. D. Caballero, A. B. Martinez, H. E. Gonzalez, O. M. Belda and V. B. Esbert, "A Novel Transition from Microstrip to a Substrate Integrated Waveguide with Higher characterisitic Impedance," Microwave Symposium Digest (IMS). Seattle,WA, pp. 1-4, June 2013.

[2] Pozar, D.M., Microwave Engineering, Third Edition, New Yourk: John Wiley \& Sons, 2005.

[3] Collin, Robert E., Foundations for Microwave Engineering, Second Edition, New Yourk: John Wiley \& Sons, 1992.

[4] Rizzi, Peter A.,Microwave Engineering, passive circuits,USA:Prentice-Hall International,Inc, 1988.

[5] J. E. Rayas-Sanchez and V. Gutierrez-Ayala,"A General EM-Based Design Procedure for Single-Layer Substrate Integrated Waveguide Interconnects with Microstrip Transitions" Microwave Symposium Digest, Atlanta,GA,pp. 983-986, June 2008. 
[6] A. A. Khan and R. Sloan,"X-Band Substrate Integrated Waveguide Power Combiner/Divider,'Microwave, Radar and Wireless Communications, Wroclaw,pp. 1-4, May 2008.

[7] X. Xu, R. G. Bosisio and Ke Wu,"A New Six-port Junction Based on Substrate Integrated Waveguide Technoloy,'IEEE Transaction on Microwave Theory And Techniques. Vol. 53, No. 7,July 2005.

[8] W. Che, K. Deng, D. Wang and Y. L. Chow," Analytical equivalence between SubstrateIntegrated Waveguide and Rectangular Waveguide" Microwave, Antennas \& Propagation, IET,pp. 35-41, February 2008.

[9] Ke Lu,"An Efficient Method for Analysis of Arbitrary Nonuniform Transmission Lines,"IEEE Trans. On Microwave theory and techniques, Vol. 45, No. 1, January 1997.

[10] Z. Hao,w. Hong, J. chen, X. chen, and K. wu," Planar diplexer for microwave integrated circuits," IEEE Proc. On Microwave Antennas and Propagat. Vol. 152, no. 6, pp. 455-459, Dec. 2005

[11] M. Bozzi, L. Perregrini, and K. Wu, "Modeling of conductor, dielectric, and radition lossrs in substrate integrated waveguide by the boundary integral-resonant mode expansion method," Microwave theory and techniques, IEEE Transactions on, vol. 56, no. 12,pp. 3153-3161,2008. 